\title{
Aerophagia Induced Reflux in Breastfeeding Infants With Ankyloglossia and Shortened Maxillary Labial Frenula (Tongue and Lip Tie)
}

\author{
Scott A. Siegel
}

\begin{abstract}
Background: Infants with tongue and possible lip tie often have a poor latch in which there is often an inadequate seal around the breast and disorganized swallowing. As a result, many of these infants swallow air during breastfeeding. Many of these infants suffer from symptoms of reflux.
\end{abstract}

Methods: This was a retrospective analysis of questionnaire/intake surveys of 1,000 infants over 5 years in private surgical practice. The inclusion of these infants was determined based on painful breastfeeding, poor lip seal, infant taking $\mathrm{H} 2$ blockers or proton pump inhibitors. These infants underwent release of their restrictions with $\mathrm{CO}_{2}$ laser.

Results: This study shows a correlation between aerophagia in infants with short maxillary labial frenula (maxillary lip tie) and ankyloglossia and reflux. A new term has been created to describe this entity: aerophagia induced reflux (AIR). Five hundred twenty (52\%) showed improvement or complete reversal of symptoms of reflux to the end point of cessation of reflux medication. Two hundred eightythree (28.3\%) had no change in reflux, and 191 (19.1\%) showed postsurgical improvement in post-feed irritability and less reflux symptoms but not successfully weaned off medications.

Conclusion: There appears to be a relationship between maxillary lip tie (ankyloglossia and shortened maxillary labial frenula) and AIR. Treatment of these infants with a relatively simple frenotomy procedure may reduce or eliminate reflux. As a result, many of these infants may be spared from invasive testing or medications that have been shown to have potentially significant side effects. This may change diagnostic and treatment algorithms.

Keywords: Aerophagia; Reflux; Ankyloglossia; Infants; Breastfeeding; Lip tie

Manuscript accepted for publication March 28, 2016

Department of Oral and Maxillofacial Surgery, Stony Brook University School of Dental Medicine, School of Medicine, State University of New York, 999 Walt Whitman Road, Suite 202 Melville, NY 11747, USA.

Email: drscottsiegel@mail.com

doi: http://dx.doi.org/10.14740/ijcp246w

\section{Introduction}

Ankyloglossia and shortened maxillary frenula (tongue and lip tie) often cause difficulty with breastfeeding. Poor latch with subsequent maternal nipple pain, poor milk transfer, failure to thrive and symptoms of reflux and colic have been described in the literature. Ultrasound studies have shown improvement in milk transfer before and after lingual frenotomy (Geddes). There have been no studies looking specifically at the phenomenon of aerophagia in these infants.

Reflux in breastfeeding infants poses many challenges for the mother-infant dyad. Aerophagia is a poorly studied but commonly seen phenomenon in breast and bottle fed infants. Aerophagia from the Greek "aerophagein" means "to eat air".

Post-feed aerophagia can be seen with higher rates in infants with ankyloglossia and possible lip tie [1-3]. When the infant attempts to latch and has an ineffective flange/seal and dysmotility of the tongue on swallowing, there may be an increase in swallowing of air, leading to post-feeding gastric distention, colic and possible reflux. This is often seen clinically in infants with ankyloglossia and/or shortened maxillary labial frenula (tongue and lip tie).

Aerophagia is often seen after feeds and is diagnosed by auscultation during feeding, presence of colic-like symptoms after feeding, and gastric distention immediately after feed and can be seen on flat plate X-ray with enlarged gastric bubble [4]. The increase in gastric pressure may overcome the lower esophageal sphincter pressure and gastric contents may reflux into the upper airway. This may be confused with other types of reflux disorders and result in misdiagnosis and improper treatment.

A review of the literature reveals three articles and one abstract concerning the relationship between ankyloglossia, lip ties, feeding problems and reflux [1-3].

Differentiating colic from gastroesophageal reflux (GER) or gastroesophageal reflux disease (GERD) is often difficult for the practitioner. In many instances, the breastfeeding history is overlooked, and the infants are empirically placed on medication or subjected to invasive endoscopic testing.

Aerophagia induced reflux (AIR) represents a different pathophysiologic mechanism compared to that of GER and/ or GERD [4].

The current lack of stringent diagnostic criteria and treatment guidelines has led to a potential misdiagnosis and/or overdiagnosis of GER or GERD. These infants are often subjected to invasive testing and/or empirically placed on medications (H2 antagonists or proton pump inhibitors (PPIs)) [6]. 
Evaluation for possible lip/tongue tie in infants with symptoms of reflux should be considered as part of the workup in diagnosis and treatment.

Recent studies have shown that PPIs have no effect on crying and irritability in infants with reflux and actually have shown an increase in upper respiratory infections and other adverse effects [6]. This may suggest an alternative cause of reflux as well [1-3].

The author is posing a potential addition to the current guidelines in diagnosis and treatment of infant reflux, especially in the breastfeeding infant. An oral examination and assessment can be performed for possible tethered oral tissues such as tongue and lip tie whose treatment may benefit the infant that is experiencing symptoms of reflux. This may result in prevention or cessation of medication that can potentially have deleterious side effects.

\section{Patients and Methods}

This was a retrospective analysis of 1,000 breastfeeding mother-infant dyads over 5 years in private oral and maxillofacial surgery practice that has a heavy emphasis on breastfeeding medicine and treatment of ankyloglossia in breastfeeding infants.

Inclusion criteria were from intake questionnaires looking for positive answers to the following questions: 1) presence of pain on breastfeeding; 2) presence of loud clicking noises during breastfeeding; 3) extended feeding times; 4) poor seal around the breast; 5) infant fussiness during and after feeding; 6) presence of reflux symptoms, especially after feed; 7) presence of post-feed gastric distention (aerophagia); 8) infant on $\mathrm{H} 2$ blockers and/or PPIs without improvement in reflux symptoms; and 9) abdominal distention immediately after feed.

Ages of infants were less than 6 months.

Clinical exam of infants was performed by the author and maxillary lip and ankyloglossia was confirmed using the Hazelbaker assessment tool for lingual frenulum function with a score of $<8$ indicating surgical intervention [7]. Kotlow classification system for maxillary labial frenulum was utilized to characterize anatomic position [8-10].

The infants underwent frenectomy/frenotomy of maxillary and lingual frenula by author with a $\mathrm{CO}_{2}$ laser of $3 \mathrm{~W}$, 1,200 mJ (novapulse LX 20, or Lightscalpel), and prilocaine/ lidocaine topical anesthesia.

Post-procedure surveys were at 1 and 2 weeks after surgery.

Inclusion consisted of answers signifying improvement from the intake questionnaire, specifically looking at reduction or elimination of reflux symptoms and weaning off or cessation of medication.

\section{Results}

Of the 1,000 infants, $526(52.6 \%)$ had an improvement of symptoms of reflux within the first week after the procedure. This was significant to the point of either reduction or cessation of H2/PPI medications. Two hundred eighty-three (28.3\%) had no change in reflux symptoms, suggesting other cause for reflux, and 191 (19.1\%) showed improvement in post-feed irritability and less symptoms of reflux but could not successfully wean off medications.

\section{Discussion and conclusion}

This study is the first to look at a larger number of subjects showing the potential linkage of aerophagia and reflux (AIR) in breastfeeding infants as it is associated with tethered oral tissues of ankyloglossia and maxillary shortened frenula. There appears to be a correlation between aerophagia associated reflux with lip and tongue tied infants.

Surgical release of these ties may help resolve these symptoms rapidly $[1,2,7,10]$.

There is a lack of literature and research looking at a potential causal relationship with aerophagia and reflux in breastfeeding infants [1-3]. It is the goal of the author to add this phenomenon to the differential diagnosis in breastfeeding infants with reflux when they are being evaluated and treated by pediatric medical providers of various specialties and subspecialties. Adding the acronym AIR and definition of AIR can assist in the differential diagnosis and management of the infant with reflux symptoms.

These infants may be spared from more invasive studies and medications that may have potentially serious side effects. The author is proposing studies to objectively look at the role of tethered oral tissue (lip and tongue ties) as a potential cause of reflux, as well as the need for a unifying classification system/validated tool for the description of lip and tongue restrictions [10]. Further studies are necessary to help establish this relationship. Also it is imperative to objectify further the combination of the tongue and lip restrictions or perhaps a single restriction that may pose the problem. Looking at possible confounding factors and the author's awareness of other causes of infantile reflux, this is still a potential cause of infantile reflux and therefore should not be overlooked and added to the differential diagnosis of practitioners who are involved in treating these infants.

This will help add to the diagnostic and treatment guidelines for reflux that are currently in use.

\section{Funding Source}

No funding was secured for this study.

\section{Financial Disclosure}

The author has no financial relationship relevant to this article to disclose.

\section{Conflict of Interest}

The author has no conflict of interest to disclose. 


\section{References}

1. Kotlow L. Infant Reflux and Aerophagia Associated with the Maxillary Lip tie And Ankyloglossia. Clinical Lactation. 2011;(2-4):25-29.

2. Siegel S. Aerophagia Induced Reflux Associated with Lip and Tongue Tie in Breastfeeding Infants. J Pediatrics. 2016;137(supplement 3).

3. Kotlow L. Infant Gastroesophageal Reflux (GER-Benign Infant Acid Reflux) or just Plain Aerophagia? International Journal of Child Health and Nutritition. 2016;5.

4. Chitkara DK, Bredenoord AJ, Wang M, Rucker MJ, Talley NJ. Aerophagia in children: characterization of a functional gastrointestinal disorder. Neurogastroenterol Motil. 2005;17(4):518-522.

5. Douglas PS. Diagnosing gastro-oesophageal reflux disease or lactose intolerance in babies who cry a lot in the first few months overlooks feeding problems. J Paediatr Child Health. 2013;49(4):E252-256.

6. Courtiol J. The cause and treatment of infant reflux. 2011.

7. Kotlow L. Oral diagnosis of abnormal frenum attachments in Neonates and infants. Journal of Pediatric Dental Care. 2004;10(3):26-28.

8. Gieruszczak-Bialek D, Konarska Z, Skorka A, Vandenplas Y, Szajewska H. No effect of proton pump inhibitors on crying and irritability in infants: systematic review of randomized controlled trials. J Pediatr. 2015;166(3):767770 e 763.

9. Hazelbaker A . Ankyloglossia: Assessment, Incidence, and Effect of Frenuloplasty on the Breastfeeding Dyad. Pediatrics. 2002;110(5):e63

10. Kotlow LA. Diagnosing and understanding the maxillary lip-tie (superior labial, the maxillary labial frenum) as it relates to breastfeeding. J Hum Lact. 2013;29(4):458-464. 